\begin{tabular}{cc}
\hline & COMPUTATIONAL RESEARCH PROGRESS IN APPLIED SCIENCE \& ENGINEERING (CRPASE) \\
& CRPASE: TRANSACTIONS OF MECHANICAL ENGINEERING \\
\hline
\end{tabular}

Research Article

\title{
Hierarchical Bayesian Based Reliability Analysis of a Sharing Storage Processor
}

\author{
Guang-Jun Jiang, Zong-Yuan Li, Hong-Xia Chen, Guan Qiao*, Hong-Hua Sun \\ School of Mechanical Engineering, Inner Mongolia University of Technology, Hohhot 010051, Inner Mongolia, China
}

\begin{tabular}{|c|c|}
\hline Keywords & Abstract \\
\hline $\begin{array}{l}\text { Hierarchical Bayesian, } \\
\text { Reliability Analysis, } \\
\text { Sharing Storage Processor. }\end{array}$ & $\begin{array}{l}\text { To tackle the actual restrictions of limited data and inaccurate reliability evaluation, a } \\
\text { hierarchical Bayesian model and multi-source information fusion joint method is } \\
\text { investigated and then implemented to the parameter estimation of failure data as well as } \\
\text { reliability analysis of a Sharing Storage Processor (SSP). Firstly, prior information in } \\
\text { counting type on the reliability of the SSP is collected. As a basis of the characteristic of the } \\
\text { Bayesian information updating, a hierarchical Bayesian framework is established by a } \\
\text { combination of the prior information obtained. Then a hierarchical Bayesian model and } \\
\text { multi-source information fusion joint method is proposed to accurately estimate the } \\
\text { parameters of failure data of the SSPs. The results of this study are validated by a } \\
\text { comprehensive comparison among the experts knowledge based technique, conventional } \\
\text { Bayesian model, as well as hierarchical Bayesian model by using the collected failure data } \\
\text { of SSPs, the results proved the feasibility of implementation of joint method to accurately } \\
\text { estimate the parameters of distributions and furtherly to analyze the reliability of products } \\
\text { with counting type failure data such as SSPs. }\end{array}$ \\
\hline
\end{tabular}

\section{Introduction}

Failure data extracted from reliability tests are always limited due mainly to the restrictions of terminate testing cost, experiment time, and accessibility of experimental facilities [1]. On the other hand, however, engineers use to turn to specialists in the field to collect objective and personal knowledge to numerically understand the features of systems/product reliability. Unfortunately, neither of the two aforementioned information sources is sufficient to model the reliability of systems/products as then a perfect and convincing reliability analysis cannot be expected [2]. Hence, analysts are trying to fuse the limited subjective test data as well as objective specialists' knowledge to model reliability of systems/products by using tools been developed according to Bayesian theory, expecting to drive an effective, accurate, economic, and feasible reliability analysis [3].

\footnotetext{
* Corresponding Author: Guan Qiao

E-mail address: jianggj_2003@163.com
}

Received: 5 May 2021; Revised: 22 May 2021; Accepted: 25 May 2021

https://doi.org/10.52547/crpase.7.2.2334
Required information by Bayesian inference such as historical information and experience knowledge often cannot match the statistical results. This inconsistency makes the reliability assessment difficult to be carried out [4]. Hence, Dong et al. [5] proposed a fuzzy fault tree method based on expert heuristics and fuzzy theory. Ben-Arieh et al. [6] proposed a group decision-making method using expert judgment according to the classification of information aggregation, which is proved to be effective for expertise polymerization. Honarmand et al. [7] used a Bayesian model based on error correlation to combine information presented in a reliability model. Li et al. [8] developed a novel multiple information fusion technique as a basis of that integrated failure data from test and that form experts' options together using Bayesian theory to conduct a comprehensive reliability analysis of a turbine blade. Zhang et al. [9] proposed a multisource information fusion method to assess the performance of control room operators. Furthermore, the Bayesian network, Markov chain, factor graph, etc. are created to solve the uncertainty of parameters of the prior information that

Please cite this article as: G. J. Jiang, Z. Y. Li, H.X. Chen, G. Qiao, H. H. Sun, Hierarchical Bayesian Based Reliability Analysis of a Sharing Storage Processor, Computational Research Progress in Applied Science \& Engineering, CRPASE: Transactions of Mechanical Engineering 7 (2021) 1-4, Article ID: 2334. 
been given in advance of the Bayesian inference. However, calculating modeling parameters of the Bayesian model always call for perfectly removing the challenges in the calculation process such as different types of data that collected [10]. Overall, the Bayesian approach provides a rigorous probabilistic framework to calibrate and update the model parameters by orderly consider objective information (prior data) as well as subjective information (testing data) [11]. However, existing Bayesian methods have been criticized for ignoring the discernibility of the estimated values observed when inferring modeling parameters from multiple data sets and which calling for a deeper model technique to accurate the parameters estimated by conventional Bayesian methods.

The hierarchical Bayesian model, as an extension of the conventional one by modeling extra parameter layers and variable parameters, is a complex Bayesian model created to confirm the accuracy of the parameters by a more detailed modeling form [12]. The benefit of more accurate analytical results of the hierarchical Bayesian model can be regarded as a consequence of a much more complex calculation process than the conventional method requires. As a consequence, several sampling methodologies like Markov chain Monte Carlo (MCMC) [13], as well as Gibbs sampling [14] methods, have been developed for reducing the computational cost when applying the mentioned methods. Hence, this paper creates a hierarchical Bayesian framework to solve the inaccurate assessment of parameters of product reliability and subsequently to complete a comprehensive reliability analysis of the object. In the study, both historical failure information, as well as expert knowledge of the SSP, are involved.

The remainder of this paper is organized as follows. The methodologies that been used in this paper including the hierarchical Bayesian model, multi-source information fusion are introduced in Section 2. The results are demonstrated in Section 3. Comparisons together with discussion are provided in Section 4. Conclusions are in Section 5.

\section{Methodology}

\subsection{Hierarchical Bayesian Model}

The hierarchical Bayesian model is a combination of hyperparameters of the prior distribution and Bayesian method, which is an extension of the traditional Bayesian model that models expert experience directly to the prior distribution. The latter always introduce considerable errors to the reliability analysis results. In the framework of Bayesian theory, all unknown parameters are considered as random variables, and the joint prior distribution of each parameter should be established. Assuming that $p(\alpha)$, $L(y \mid \alpha)$, and $q(\alpha \mid y)$ are prior distribution, likelihood function, and posterior distribution, respectively. $\alpha$ is the parameter to be estimated. According to Bayesian theory, the posterior parameter can be inference by

$q(\alpha \mid y) \propto p(\alpha) L(y \mid \alpha)$
For a prior distribution with a likelihood function, see in Figure 1, the Bayesian model can be expressed as

$$
f(b \mid y) \propto f(y \mid b) f(b \mid a)
$$

Accordingly, the hierarchical model, as shown in figure 2 , can be similarly expressed as

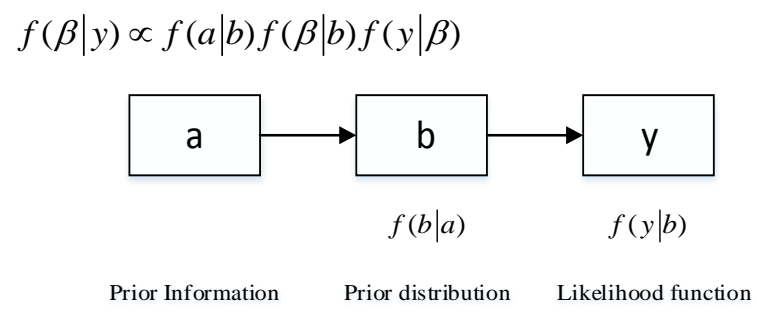

Figure 1. Single-layer Bayesian model

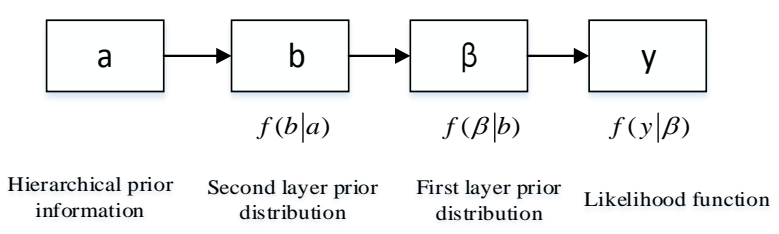

Figure 2. Two-layer Bayesian model

An arbitrary parameter $(\alpha)$ of a distribution can be further updated by a given data set $\mathrm{D}$, consequently, the posterior distribution can be expressed as

$$
q(\alpha, y \mid D) \propto p(\alpha \mid y) L(D \mid \alpha, y)
$$

The more information such as experts' knowledge introduced into information updating the more accurate results will be obtained.

The average value of all the prior information can be computed by

$T\left(p 1, p 2, p 3 \cdots, p_{n}\right)=\sum_{i=1}^{n} \alpha_{i} p_{i}$

The geometric merge method can be used to multiply each prior information to fuse the prior information by

$T\left(p 1, p 2, p 3 \cdots, p_{n}\right)=\prod_{i=1}^{n} p_{i}^{\alpha_{i}}$

where, $p_{i}$ is single prior information, $\alpha_{i}$ represents the weight of each priority information and $\sum_{i=1}^{n} \alpha=1, T$ denotes fused prior information.

The prior information can be transformed into a linear distribution form by

$\log T\left(p 1, p 2, p 3 \cdots, p_{n}\right)=\sum_{i=1}^{n} \alpha_{i} \log p_{i}$

Eqs. (5) to (7) can effectively fuse multiple prior information of deterministic Bayesian models.

\subsection{Likelihood Functions}

The logistic regression model is an effective tool to reduce the deviations of the estimated parameters. Assume that a dependent variable has $\mathrm{m}$ influence factors denoted as 
$\alpha, x_{1}, x_{2}, \ldots x_{m}$. According to the conditional probability $P\left(y=1 \mid x_{i}\right)=p_{i}$, the logistic regression model can be constructed as

$$
\begin{aligned}
\log i t\left(p_{i}\right) & =\ln \left(\frac{p_{i}}{1-p_{i}}\right) \\
& =\alpha+\beta_{1} x_{1}+\cdots+\beta_{m} x_{m} \\
& =\alpha+\sum_{j=1}^{m} \beta_{j} x_{j}, j=1,2, \ldots, m
\end{aligned}
$$

By quantifying the factors affecting the reliability of products and constructing a logistic probability distribution function, information from different experts can be effectively integrated. A probability model can be obtained as

$$
p_{i}=F\left(y_{i}\right)=F\left(\alpha+\beta x_{i}\right)=\frac{1}{1+e^{-y_{i}}}=\frac{1}{1+e^{-\left(\alpha+\beta x_{i}\right)}}
$$

In which, $p_{i}$ represents the probability, $F\left(y_{i}\right)$ is the cumulative density function for a given $x_{i}, p_{i}$ indicates the probability of the corresponding individual influences overall population, $y_{i}$ is denotes hidden variables that can be converted to probability via a logistic function.

Additionally, the Metropolis-Hastings algorithm is a simple but effective numerical simulation algorithm used to obtain a random sample from the posterior distribution [17]. Parameter estimation methods used to employ the Metropolis-Hastings algorithm to determine the accuracy of a candidate value. Considering that the assessment of the parameters in reliability engineering sometimes turn to experts' experiences in the field and subsequently induced unneglectable subjective to the assessment results $[17,18]$, hence, the MCMC method is considered as an accurate tool to stabilize the results through multiple iterations of the parameter estimation of reliability analysis by using heretical Bayesian technique. The procedure of conducting the MCMC method for generating posterior distribution $f(\theta \mid y)$ with the sampling size of $\mathrm{N}$ is as follows. Note that $\theta^{t}$ is the generating value at $t$ th iteration.

(i) Set initial candidate points $\theta^{0}$. When the parameter vector element is to be connected, $q\left(\theta^{*} \mid \theta\right)$ is referred to as a suggested density function which is used for generating $\theta$ from $\theta^{*}$.

(ii) For $n=1, \ldots, N$ repeats the following steps: set $\theta=\theta^{n-1}$ ; generate new candidate values of $\theta^{*}$ from the suggested density $q\left(\theta^{*} \mid \theta\right)$; calculate the acceptance probability $\alpha$ which refers to the probability that a candidate point is accepted as the next simulation point, expressed as

$$
\alpha=\min \left(1, \frac{f\left(\theta^{*} \mid y\right) q\left(\theta \mid \theta^{*}\right)}{f(\theta \mid y) q\left(\theta^{*} \mid \theta\right)}\right)
$$

(iii) After calculating the acceptance probability, generating a random number that obeys the uniform distribution $(0,1)$ and compares it with $\alpha$. If the random number is less than $\alpha$, the candidate point is accepted, and the updated point is recorded as $\theta^{t}=\theta^{*}$, otherwise, the candidate points are rejected, and the result returns to the previous one.

This algorithm does not need to evaluate the normalization constants $f(y)$ contained in the posterior distribution $f(\theta \mid y)$ one by one, hence, the acceptance probability can be simplified as

$$
\alpha=\min \left(1, \frac{f\left(\theta^{*} \mid y\right) f\left(\theta^{*}\right) q\left(\theta \mid \theta^{*}\right)}{f(\theta \mid y) f(\theta) q\left(\theta^{*} \mid \theta\right)}\right)
$$

Table 1. Fault data have been applied in the study

Test environment

Failures

15142313644423224552532231125141112132535251152

According to the features of the data as well as specialists' knowledge, a Gamma model (with prior parameters of $\alpha_{v}=5$ and $\beta_{k}=1$ ) were selected for modeling the lifetime the those SSPs. A mature software namely OpenBUGS was implemented for parameter simulations. The posterior probability density function of $\alpha$ obtained, see Figure 3, after 50,000 iterations.

Similarly, the posterior probability density function of $\beta$ obtained, see Figure 4, after the same amount of iteration.

The updated parameters $\alpha$ and $\beta$ after convergence are shown in Table 2.

In Figure 5, $\alpha_{0}, \alpha_{1}, \alpha_{2}$ are affecting parameters, which generally represented by normal distribution or uniform distributions in the case of insufficient data. The prior distribution of parameters in this study are designed as follows

$$
\begin{aligned}
& \alpha_{0} \sim \operatorname{dnorm}(0,0.001), \\
& \alpha_{1} \sim \operatorname{dnorm}(0,0.001), \\
& \alpha_{2} \sim \operatorname{dnorm}(0,0.001), \\
& \tau \sim \operatorname{dgamma}(7.691,1.364)
\end{aligned}
$$

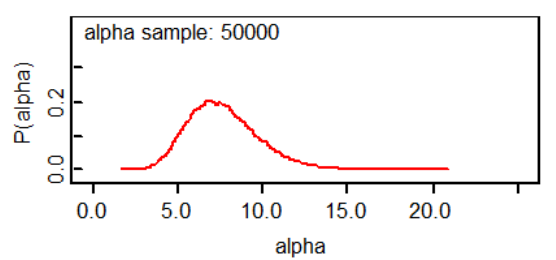

Figure 3. Posterior distribution probability density function of $\alpha$ 


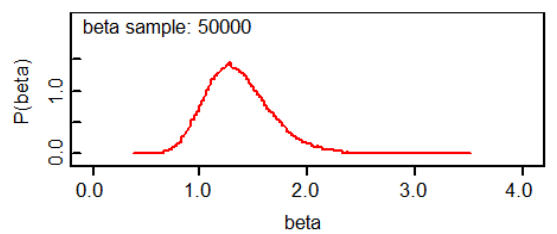

The MCMC algorithm was used to simulate the output of the above model. The simulation results of the parameters are shown in figure 6. As shown in the Table 3, MC errors are close to zero which indicates the selected model matches the parameter distribution.

Figure 4. Posterior distribution probability density function of $\beta$

Table 2. The updated parameters $\alpha$ and $\beta$.

\begin{tabular}{cccccc}
\hline \multirow{2}{*}{ Parameters } & \multicolumn{3}{c}{ Statistical Features } & \multicolumn{2}{c}{ Posterior Confidence Interval } \\
\cline { 2 - 6 } & Mean & SD & MC-error & $2.5 \%$ & $97.5 \%$ \\
\hline$\alpha$ & 7.691 & 2.085 & 0.02945 & 4.254 & 12.38 \\
$\beta$ & 1.364 & 0.3016 & 0.004115 & 0.8607 & 2.046 \\
\hline
\end{tabular}

Table 3. The simulation results of parameters

\begin{tabular}{cccccc}
\hline \multirow{2}{*}{ Parameters } & \multicolumn{3}{c}{ Statistical } & \multicolumn{2}{c}{ Posterior Confidence Interval } \\
\cline { 2 - 6 } & mean & SD & MC-error & $2.5 \%$ & -2.665 \\
$\alpha_{0}$ & -2.97 & 0.161 & 0.001188 & -3.289 & 44.26 \\
$\alpha_{1}$ & 0.174 & 22.19 & 0.1129 & -42.99 & 43.32 \\
$\alpha_{2}$ & 0.1574 & 22.19 & 0.1127 & -43.86 & 12.88 \\
$\tau$ & 8.059 & 2.168 & 0.01795 & 4.471 & \\
\hline
\end{tabular}

Based on the parameters that been influenced by hierarchical Bayesian analysis, the reliability of the SSPs is further analyzed. The results show that the life of the SSPs are affected by temperature, humidity, and human operations. Moreover, the impact of these factors cannot be ignored for the long-life, high reliability, and safe operation requirements. To this end, a piece of multi-source information was conducted to fuse various information related to the reliability of the SSPs. The information fusion framework is shown in Figure 5.

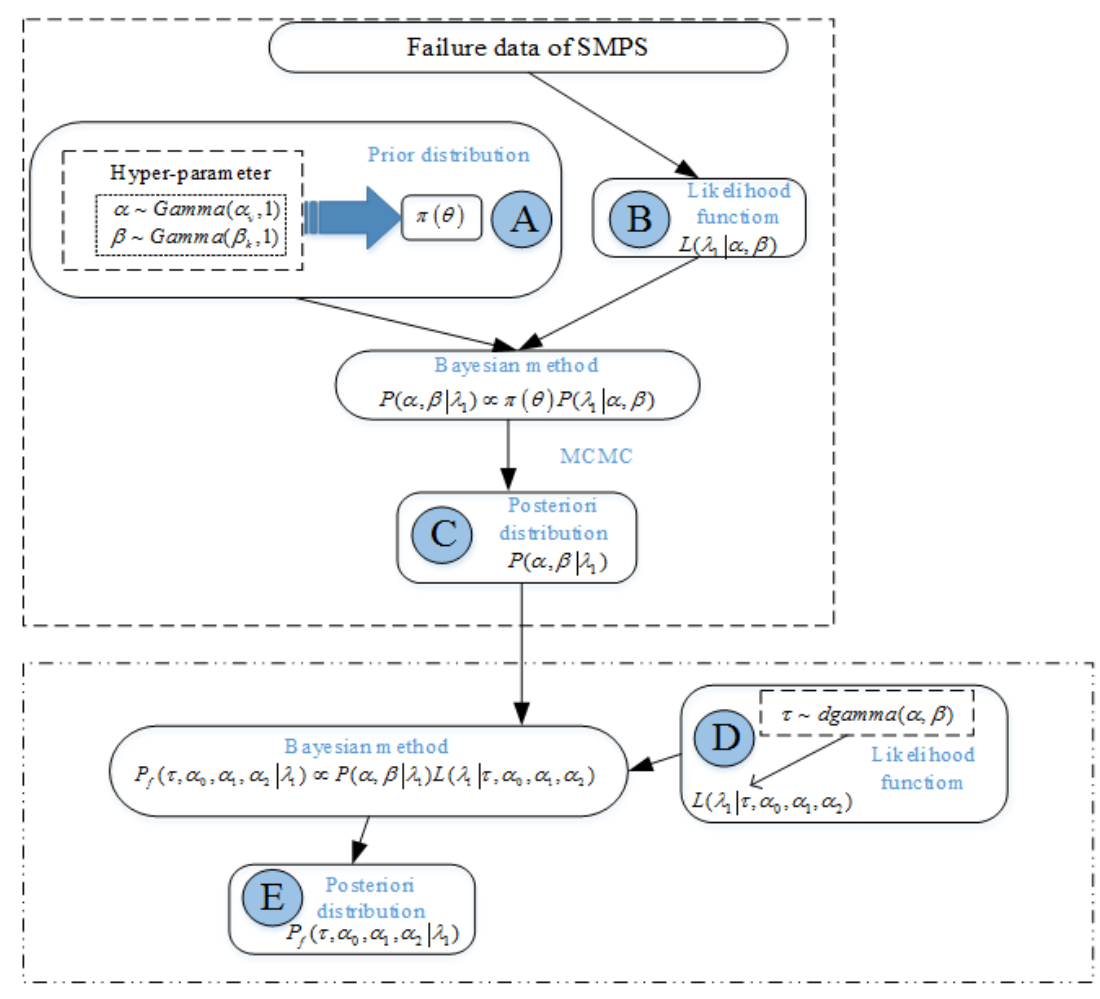

Figure 5. Hierarchical Bayesian framework to fuse multi-source information of SSPs 


\section{Comparisons and Discussions}

In this section, a comparison analysis of predicted failures using the conventional Bayesian model, proposed a hierarchical Bayesian framework, as well as expert knowledge, are carried out to validate the results of this study. Specifically, the comparison between expert experience and the conventional Bayesian model is shown in Figure 6. The comparison between the results of the conventional Bayesian model and the proposed hierarchical Bayesian framework is demonstrated in Figure 7. The comparison between the results of the hierarchical Bayesian framework and hierarchical Bayesian framework considering multi-source information fusion is displayed in Figure 8.

The Bayesian method can be applied well in the analysis of small sample reliability estimation. In this paper, by establishing a hierarchical Bayesian framework for the reliability analysis of SMPs of a Blue Mountains supercomputer at Los Alamos National Laboratory was concluded. figure 6 indicates that the reliability and lifetime parameters of SMPs are difficult to determine according to only to the data collected from experts. However, the same figure also demonstrated that the confidence interval of the parameter of the life of the SSPs is significantly reduced after the conventional Bayesian model was implemented. Due to data limitation, the hierarchical Bayesian model can further decrease the confidence interval of the parameter of life of the SSPs than the conventional Bayesian model, which is shown in Figure 7. By the end, multi-source information fusion is considered to integrate the information from various sources and as a basis of that to combine several prior distributions by a weighted algorithm. And accordingly, more accurate parameter estimation of lifetime of the SSPs is concluded, see Figure 8. The comparisons indicated that the proposed hierarchical Bayesian model and multi-source information fusion joint method is good at estimate parameter estimation of a lifetime more accurate than exciting methods.

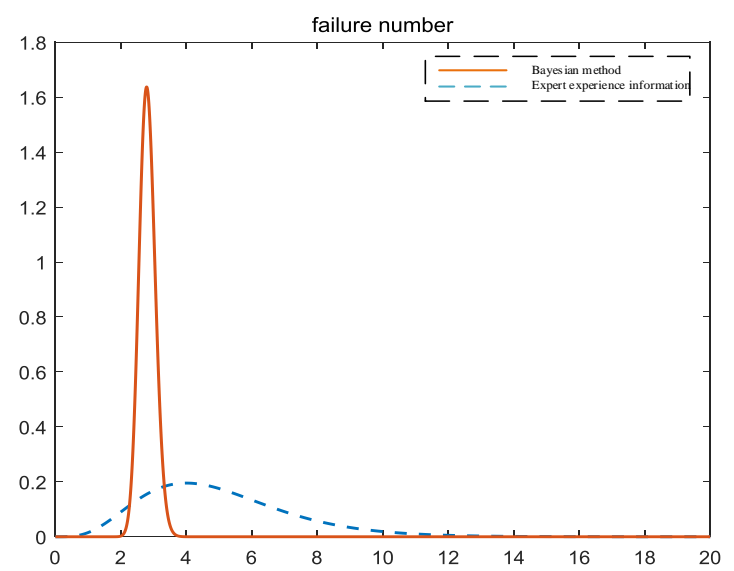

Figure 6. The comparison between expert experience and the conventional Bayesian model

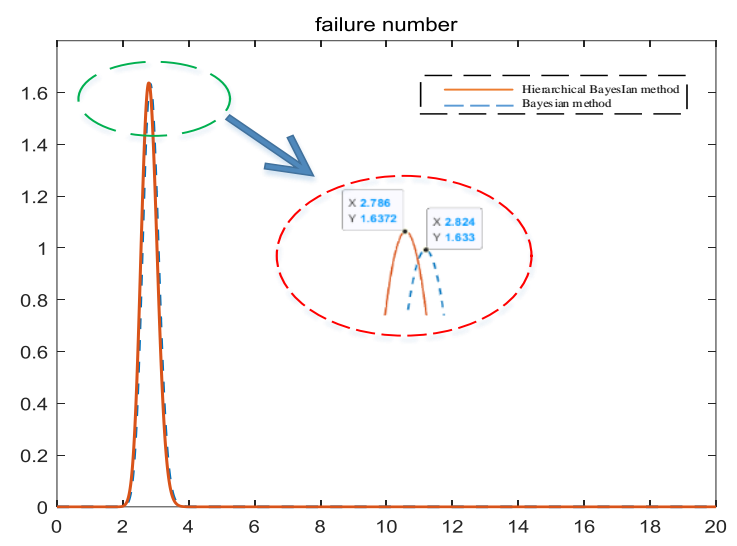

Figure 7. The comparison between the conventional Bayesian model and proposed a hierarchical Bayesian framework

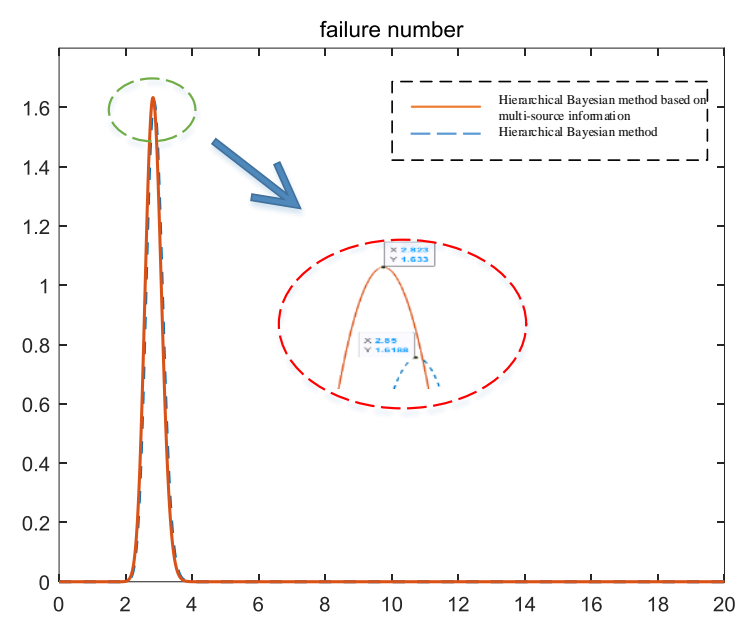

Figure 8. The comparison between the hierarchical Bayesian framework with and without considering multi-source information fusion

\section{Conclusions}

For overcoming the limitation that failure data are always insufficient to determine their distribution parameters of SSPs, a hierarchical Bayesian model, and multi-source information fusion joint method was proposed as a basis of that to complete the parameter estimation of distributions and accordingly to conclude reliability analysis of the SSPs. A comparison analysis of predicted failures using the conventional Bayesian model, proposed hierarchical Bayesian framework, as well as expert's knowledge, were carried out to validate the results of this study and the results of the comparison indicates the feasibility and creativeness of the proposed method, additionally, the advancements of the proposed method that much more accurate than conventional Bayesian model, proposed hierarchical Bayesian framework, as well as expert's knowledge-based methods. And it should be highlighted according to the results of this study the hierarchical Bayesian model and multi-source information fusion joint method are applicable for estimation of the reliability of the product when the testing data is insufficient. 


\section{Acknowledgements}

This work was partially supported by National Natural Science Foundation of China under the contract number 71761030 and 51965051, the Natural Science Foundation of Inner Mongolia under the contract number 2019LH07003.

\section{Conflict of Interest Statement}

The authors declare no conflict of interest.

\section{References}

[1] H. Li, , C. Guedes Soares, H.Z. Huang, Reliability analysis of a floating offshore wind turbine using Bayesian Networks, Ocean Engineering 217 (2020) 107827.

[2] R.P. de Oliveira, J.A. Achcar, D. Peralta, J. Mazucheli, Discrete and continuous bivariate lifetime models in presence of cure rate: a comparative study under Bayesian approach, Journal of Applied Statistics 46 (2019) pp 449-467.

[3] H. Li, A.P. Teixeira, C. Guedes Soares, A two-stage Failure Mode and Effect Analysis of offshore wind turbines, Renewable Energy 162 (2020) 1438-1461.

[4] W. Choi, B.D. Youn, H. Oh, N.H. Kim 2, A Bayesian approach for a damage growth model using sporadically measured and heterogeneous on-site data from a steam turbine, Reliability Engineering \& System Safety 184 (2019) 137-150.

[5] Y.H. Dong, D.T. Yu, Estimation of failure probability of oil and gas transmission pipelines by fuzzy fault tree analysis, Journal of loss prevention in the process industries 18 (2005) pp 8388.

[6] D. Ben-Arieh, Z. Chen, Linguistic group decision-making: opinion aggregation and measures of consensus, Fuzzy Optimization and Decision Making (2006) 371-386.

[7] P. Honarmandi, T.C. Duong, S.F. Ghoreishi, D. Allaire, R. Arroyave, Bayesian uncertainty quantification and information fusion in CALPHAN-based thermodynamic modeling, Acta Materialia 164 (2019) pp 636-647.

[8] H. Li, H.Z Huang, Y.F. Li, J. Zhou, J. Mi, Physics of failurebased reliability prediction of turbine blades using multi- source information fusion, Applied Soft Computing 72 (2018) 624-635.

[9] X.G. Zhang, S.K. Mahadevan, N. Lau, M.B. Weinger, Multisource information fusion to assess control room operator performance, Reliability Engineering \& System Safety 194 (2020) 106287.

[10] S. Sankararaman, S. Mahadevan, Likelihood-based representation of epistemic uncertainty due to sparse point data and/or interval data, Reliability Engineering \& System Safety 96 (2011) 814-824.

[11] E. Vanderhorn, S. Mahadevan, Bayesian model updating with summarized statistical and reliability data, Reliability Engineering \& System Safety 172 (2018) 12-24.

[12] H. Li, C. Guedes Soares, Reliability analysis of floating offshore wind turbines support structure using hierarchical Bayesian network, In Proceedings of the 29th European Safety and Reliability Conference (2019) 2489-2495. Research Publishing Services Singapore.

[13] J.B. Nagel, B. Sudret, Hamiltonian Monte Carlo and borrowing strength in hierarchical inverse problems, ASCEASME Journal of Risk and Uncertainty in Engineering Systems, Part A: Civil Engineering 2 (2015) B4015008.

[14] J.B. Nagel, B. Sudret, Bayesian multilevel model calibration for inverse problems under uncertainty with perfect data, Journal of Aerospace Information Systems 12 (2015) pp 97 113.

[15] J. Guo, H. Zheng, B. Li, G.Z. Fu, Bayesian Hierarchical Model-Based Information Fusion for Degradation Analysis Considering Non-Competing Relationship, IEEE Access 7 (2019) 175222-175227.

[16] Y. Huang, J.L. Beck, H. Li, Hierarchical sparse Bayesian learning for structural damage detection: Theory, computation and application, Structure Safety 64 (2017) 37-53.

[17] W.K. Hastings, Monte Carlo sampling methods using Markov chains and their applications, Biometrika 57 (1970) 97-109.

[18] H. Li, H. Diaz, C. Guedes Soares, A developed failure mode and effect analysis for floating offshore wind turbine support structures, Renewable Energy 164 (2021) 133-145. 